
\title{
28 Research Square \\ Performance of etched silica FBG for simultaneous strain temperature measurement
}

\section{Koustav Dey}

NIT Warangal: National Institute of Technology Warangal

\section{Ramesh Buddu}

Institute for Plasma Research

\section{Sourabh Roy ( $\nabla$ sroy@nitw.ac.in )}

National Institute of Technology Warangal https://orcid.org/0000-0002-8034-7367

\section{Research Article}

Keywords: Fiber Bragg gratings (FBGs), Chemical etching, Interrogation technique, Strain measurement, Temperature measurement.

Posted Date: April 16th, 2021

DOl: https://doi.org/10.21203/rs.3.rs-424871/v1

License: (c) (i) This work is licensed under a Creative Commons Attribution 4.0 International License. Read Full License 


\section{Abstract}

In this article, we have extensively investigated the efficiency of etched silica-based fiber Bragg gratings (FBGs) for the experimental measurement of temperature and strain simultaneously. A single-multi-single mode fiber (SMS) combination is employed in the circuitry which acts as a bandpass filter for efficient interrogation technique. We recorded the sensitivity of $20.30 \mathrm{pm} /{ }^{\circ} \mathrm{C}$ and $2.89 \mathrm{pm} / \mu \rrbracket$ over the temperature and strain range of $25-225^{\circ} \mathrm{C}$ and $50-2050 \mu \rrbracket$, respectively. These data were recorded with the strain resolution of $\pm 10 \mu \rrbracket$ whereas the resolution for the temperature is $\pm 0.50^{\circ} \mathrm{C}$. The experimental results show the potentiality of the sensor for measuring the strain and temperature simultaneously with higher intrinsic sensitivity and resolution.

\section{Introduction}

Fiber optic sensors (FOSs) have been come up as a promising sensing element owing to their unique features over other conventional sensors for various applications in the last few decades [1-4]. Measuring different parameters simultaneously mostly strain and temperature in many practical applications became research hotspot for more than a decade. However, crosstalk is the main issue in case of measuring several parameters simultaneously, which is undesirable in common sensing applications. So far, numerous configurations are available and they are investigated to address the issue of cross-sensitivity in the case of simultaneous measurement of strain and temperature. Several intrinsic optical devices such as Polarization maintaining (PM) fiber [5-6], Fabry-Perot (FP) [7-10], long-period gratings (LPGs) [11-12] are incorporated with FBG based sensing techniques to offer possible solutions for cross-sensitivity effect. Employing PM fiber will be expensive for a common fiber sensing system. Additionally, inscribing an FBG in a PM fiber is a robust and efficient method for strain-temperature measurement simultaneously. FP based techniques could be employed for high-temperature measurement along with high-resolution detection. But the main drawback is in the fabrication which needs complicated and expensive process including the laser-micromachinery technique. Furthermore, laser-irradiated fiber is significantly more fragile, attributed to fiber profile deformation. Recently, Dan Su et al. [13] proposed compact dual FBGs inscribed over SMF, thin core fiber (TCF) and the interface of them with splicing technology by high peak power femtosecond laser to measure strain and temperature simultaneously. This scheme has its limitations due to being expensive and complicated fabrication technique. Also achieving special resonance at the splicing point is not so easy task. An FBG sensor embedded with an SMS has been reported for measuring the temperature and strain with higher accuracy [14]. But the main bottleneck of this technique is to have the equivalent sensitivity of both the components, which is quite difficult to achieve.

For certain applications, a miniaturized sensor with higher intrinsic sensitivity is required. Therefore, any technique that offers miniaturization and the capability of simultaneous measurement will be highly promising than previously reported techniques. Z. Kang et al. [15] demonstrated an up-taper based MachZehnder interferometer technique for simultaneous measurement. Although this technique offers higher temperature sensitivity, it limits due to a special and precise fabrication technique and the small strain 
sensitivity $(0.74 \mathrm{pm} / \mu \mathbb{\mathrm { Q }})$. Q. Liu et al. [10] integrated an FBG and FP for simultaneous measurement. This scheme has its limitations due to its high cost and complicated fabrication process. Although LPG based method [12] offers higher sensitivity than other photonic sensors, the main bottleneck in using LPG is cladding mode nature which makes it more undefended to the external perturbations like bending and/or temperature imbalance in the encompassed medium, which affects the performance of the sensor system.

Factitively, an etched FBG can be employed for this purpose due to its simpler fabrication approach, lower loss, miniaturized size and absence of frail splicing point, which made it more attractive towards the aforesaid applications. Sridevi. S et al, [16] have proposed an etched FBG based sensor with enhanced sensitivity and higher measurement range.

In this paper, an upgraded sensor design with higher sensitivities and measurement range compare to the previously reported methods is proposed using etched silica FBG. An edge filtering technique using an optimized SMS has also been included. Using the proposed etched silica-based edge-filtering method, the capability for simultaneous quantification of strain and temperature has been demonstrated. It shows a higher sensitivity for temperature and strain of $20.30 \mathrm{pm} /{ }^{\circ} \mathrm{C}$ and $2.89 \mathrm{pm} / \mu \otimes$, respectively. A comprehensive comparative table has been provided to emphasize the performance of our proposed method with respects to the other similar reported methods.

\section{Experimental Set-up And Fabrication Details}

The configuration of the present sensor is given in Fig. 1 .

As shown in Fig. 1, the proposed experimental set-up consists of a super-luminescent light-emitting diode (SLD, 1300-1800 nm) as the light source, a circulator, SMS integrated with an FBG, an optical spectrum analyser (OSA, Agilent 86142B) as a detector whose wavelength resolution is $\sim 10 \mathrm{pm}$, an oven ( 0 to $250^{\circ} \mathrm{C}$ ) connected with a dimmer-stat and a one-dimensional micro-translational stage with the minimum adjustable displacement of $0.01 \mathrm{~mm}$. A calibrator thermometer (CL3515R, OMEGA) was kept inside the oven for accurate measurement of the temperature.

At first, light from the SLD source will be coupled to the FBG and will get reflected. The reflected light from the FBG will be filtered by the SMS and reaches the detector through the circulator.

\subsection{Design of SMS fiber structure}

The SMS fiber structure can be constructed by sandwiching an optimized section of the multi-mode fiber between two single-mode fibers. All the fiber sections are in-line so that there is no offset between the fiber interfaces. The underlying principle of such spliced fiber is the multi-mode interference (MMI). As the MMF length is the main factor contributing to the different slopes' regions of the spectrum, hence the MMF length has been optimized as described in our previous work [17]. One of the slope edges of the spectrum has been chosen as per the requirement and employed in the proposed technique. 
In our proposed method, such SMS structure has been used for an efficient edge filtering technique for measuring the temperature and strain simultaneously.

\subsection{Preparation of etched silica FBG}

The etching was performed on the silica-based FBG using hydrofluoric acid. The comprehensive chemical reaction in this process is given by:

$\mathrm{SiO}_{2}+6 \mathrm{HF}=2 \mathrm{H}^{+}+\mathrm{SiF}_{6}{ }^{2-}+2 \mathrm{H}_{2} \mathrm{O}$

The etching rate solely depends on the concentration of the solution and the temperature.

To control the etching of the FBG inscribed in the SMF-28e (corning) fiber in an effective way, firstly the etching rate evaluated using the SMF-28e fiber. For this purpose, we took a dozen samples were immersed in a $40 \% \mathrm{HF}$ solution, taken out every 5 minutes and the diameter of the etched section in various positions were measured by optical microscopy after careful cleaning and drying. It is found that the etching rate is $1.61 \mu \mathrm{m} / \mathrm{min}$ at $25 \pm 1^{\circ} \mathrm{C}$ (room temperature). To fully etched the FBG of length $1 \mathrm{~cm}$, the FBG is dipped into $40 \% \mathrm{HF}$ solution to reduce its cladding diameter to around $10 \mu \mathrm{m}$, which has been optimized to sustain an above-mentioned range of strain and just to get the effective and stable "Evanescent field wave (EFW)" to improve the sensing quality of the FBG probe.

An inverted optical microscope was used to confirm the effectiveness of the etching throughout the length of the grating. The obtained images are shown in Fig. 2. Figure 2(a) depicts the image for the unetched FBG whereas the etched FBG with $10 \mu \mathrm{m}$ thickness of the fiber is shown in Fig. 2 (b).

By removing the cladding width adequately, the EFW gets strengthen which in turns increases the device sensitivity [18].

\section{Results And Discussions}

In this section, the experimental validation for simultaneous measurement has been carried out.

To determine the temperature co-efficient, a temperature change was applied over the range of $25^{\circ} \mathrm{C}$ to $225^{\circ} \mathrm{C}$ with an interval of $5^{\circ} \mathrm{C}$ (at zero strain) onto the etched grating. The change of the optical power was monitored using the OSA. Similarly, the peak wavelength shift of the FBG peak was monitored and noted down during the change of the temperature over the aforementioned range.

To evaluate the strain co-efficient, the applied strain was increased from $50 \mu \rrbracket$ to $2050 \mu \rrbracket$ with an interval of $110 \mu \Downarrow$ at a constant temperature $\left(\sim 23^{\circ} \mathrm{C}\right)$. The shift of the Bragg wavelength of the FBG peak due to applied strain was also monitored.

The results are shown in Fig. 3. Good linearity was observed for all the measurements. Figure 3 (a) \& (b) present the linear fittings of variation of the power loss at the time of applying the temperature and strain individually at zero strain and $23{ }^{\circ} \mathrm{C}$ of temperature respectively. Whereas, Fig. 3 (c) \& (d) depicts the 
wavelength shift of the FBG upon the application of the temperature and strain over the range of 25$225^{\circ} \mathrm{C}$ of temperature and $50-2050 \mu \otimes$ of strain at zero strain and constant room temperature, respectively.

With increasing the temperature, a redshift can be observed in the spectrum due to the increase in the refractive index of the cladding as well as the core mode. For finding temperature sensitivity, the wavelength shifts during rising as well as for lowering the temperature has taken into consideration. In our proposed method, the measurement in temperature and strain is restricted due to the measurement limit of the instruments.

From the experimental result it can be inferred that, at the time of increasing as well as decreasing the temperature, there is no evidence of the hysteresis due to the high repeatability and overlapping of the data. The FBG peak represents redshift which is expected due to the thinning of the fiber. As the etched FBG became thinned, the period of the grating affects more than the unetched FBG sensor, which in turns enhance the sensitivity for the etched-FBG sensor. The higher temperature sensitivity is due to the decrease in the thermo-optic coefficient of the thinned Grating.

Now, the well-known transfer matrix can be fitted by applying linear-fit to each measured characteristic in Fig. 3. Here the coefficient is related to the measurement of the power and wavelength shift with external perturbations [19]:

$$
\left[\frac{\Delta T}{\Delta \varepsilon}\right]=\frac{1}{D}\left[\begin{array}{cc}
K_{\varepsilon P} & -K_{\mathrm{\varepsilon} \lambda} \\
-K_{T P} & K_{T \lambda}
\end{array}\right]
$$

Where the determinant is $D=K_{T P}{ }^{*} K_{\mathrm{\varepsilon} \lambda}-K_{\mathrm{\varepsilon} P}{ }^{*} K_{T \lambda}$; Where $K_{T P}, K_{\mathrm{\varepsilon} \lambda}, K_{\mathrm{\varepsilon} P}$ and $K_{T \lambda}$ are the matrix coefficients for power/temperature, wavelength/strain, power/strain and wavelength/temperature.

$$
\left[\frac{\Delta T}{\Delta \varepsilon}\right]=\frac{1}{1.68}\left[\begin{array}{cc}
-0.077 \mathrm{~dB} / \mu \varepsilon & 2.89 \mathrm{pm} / \mu \varepsilon \\
0.93 \mathrm{~dB} /{ }^{\circ} \mathrm{C} & 20.30 \mathrm{pm} /{ }^{\circ} \mathrm{C}
\end{array}\right]\left[\frac{\Delta \lambda}{\Delta P}\right]
$$

The obtained higher value of the matrix co-efficient overcomes the problem of noise sensitiveness [20].

The achieved temperature sensitivity of the proposed sensor $20.30 \mathrm{pm} /{ }^{\circ} \mathrm{C}$, is roughly double to the theoretical value (w.r.to the FBG with reflected Bragg wavelength value $\sim 1550 \mathrm{~nm}$ ) i.e., $\sim 10 \mathrm{pm} /{ }^{\circ} \mathrm{C}$, whereas, the strain sensitivity of the FBG is $2.89 \mathrm{pm} / \mu \rrbracket$, which is 2.45 times higher compared to the theoretical value i.e., $\sim 1.2 \mathrm{pm} / \mu \rrbracket$.

\section{Performance Analysis Of The Designed Sensor}

To analyse the performance of this proposed scheme for simultaneous measurement, the sensor head underwent strain variations up to $1806 \mu \varepsilon$ at $300 \mu \varepsilon$ step interval at a constant temperature $\left(T=90^{\circ} \mathrm{C}\right)$. Similarly, the temperature was varied over the range $35^{\circ} \mathrm{C}-185^{\circ} \mathrm{C}$ at $30^{\circ} \mathrm{C}$ intervals for a specific applied strain $(\nabla=606 \mu \rrbracket)$. Putting the values of the wavelength shift exhibited by the sensor head using Eq. (2), 
temperature and strain changes are calculated. The results are shown in Fig. 4. The deviation with respects to the applied values have shown here.

Sensor's outputs for a particular temperature and strain are shown in Fig. 4. Figure 4(a) depicts the strain changes over the range $0-1806 \mu \rrbracket$ at $90^{\circ} \mathrm{C}$ while Fig. $4(\mathrm{~b})$ shows the variation of the temperature over the range $35-185^{\circ} \mathrm{C}$ at $606 \mu \rrbracket$.

From these plots, the maximum deviation for temperature and strain are $\pm 0.7^{\circ} \mathrm{C}$ and $\pm 32 \mu \rrbracket$ respectively.

\section{Performance Metrics Of The Proposed Sensor}

Performance metrics of the proposed sensor system such as a limit of detection (LoD) and resolution are obtained for the etched FBG based temperature and strain sensor.

\subsection{Limit of Detection:}

LoD indicates the lowest change in input that could be measured with a 3-sigma $(3 \sigma)$ marginal error. It is calculated as [21]

$$
\begin{aligned}
\text { LoD }=\frac{3 \times \text { Resolution of the Detector Unit }}{\text { Sensor's Sensitivity }} & =\frac{3 \times 0.01 \mathrm{pm}}{20.30 \mathrm{pm} /{ }^{\circ} \mathrm{C}}=0.00148{ }^{\circ} \mathrm{C} \text { (for temperature) } \\
& =\frac{3 \times 0.01 \mathrm{pm}}{2.89 \mathrm{pm} / \mu \mathrm{\varepsilon}}=0.0873 \mu \varepsilon \text { (for strain) }
\end{aligned}
$$

\subsection{Resolution:}

In order to assess the resolution of the proposed sensor for measuring the temperature and strain simultaneously, the following experiment was performed as shown in Fig. 5. For this, at first, the temperature and strain of $40{ }^{\circ} \mathrm{C}$ and $206 \mu \rrbracket$, respectively were applied to the sensor head and the respective wavelength change due to the applied temperature and strain were measured. After that, the temperature was increased to $45^{\circ} \mathrm{C}$ at a $206 \mu \rrbracket$ and the strain was changed to $406 \mu \rrbracket$ and the shift in the wavelength was measured in each case. Lastly, incorporating the values into transfer matrix Eq. (2), one can calculate the wavelength shifts due to their respective temperature and strain randomly, and measure simultaneously.

From these results, resolution of $\pm 0.5^{\circ} \mathrm{C}$ and $\pm 10 \mu \rrbracket$ were determined for temperature and strain measurements, respectively. These achieved values are limited by the resolution of the OSA, control accuracies of the oven and the cantilever set-up.

To emphasize the performance of our proposed sensor, a comprehensive comparison with different schemes has been made for measuring the temperature and strain simultaneously with respects to the different sensing parameters (Table 1). It's worth noting that the efficiency in terms of the sensing parameters of the designed sensor head is better than other similar techniques reported so far. 


\section{Conclusions}

In conclusion, an experimental study of an etched silica FBG sensor for measuring strain and temperature simultaneously has been proposed and demonstrated with higher intrinsic sensitivities and accuracy using OSA. An effective interrogation technique has been employed in the circuitry for this purpose.

The temperature and strain sensitivity were measured to be $20.30 \mathrm{pm} /{ }^{\circ} \mathrm{C}$ and $2.89 \mathrm{pm} / \mu \rrbracket$, which is double the typical values. Also, the proposed sensor has good resolution with $\pm 10 \mu \nabla$ and $\pm 0.5^{\circ} \mathrm{C}$ for strain and temperature respectively. The experimental results provide a shred of strong evidence about the sensing performance of our proposed sensor with enhanced sensitivity compared to the other reported methods. The proposed sensor has good characteristics in the applications for biochemical, biomedical due to its miniaturized size and high intrinsic sensitivity. The main drawback of the proposed sensor structure is directly related to the weakening of the sensor probe due to the smaller diameter of the fiber. This aspect can be easily addressed by adopting a proper packaging design. Further improvement of the sensor performance will be the research focus, so it can work over a wider measurement range and with enhanced higher sensitivity.

\section{Declarations}

\section{Authors' contributions}

Koustav Dey has conceptualised and performed the experiments, collected the raw data, did the primary analysis and prepared the manuscript. B. Ramesh has finalized the contents of the manuscript. Sourabh Roy has analysed the results, drawn the conclusions and finalized the contents of the manuscript.

\section{Acknowledgements}

We also thank the Board of Research in Nuclear Sciences (BRNS, DAE) for their funding.

\section{Authors Declarations:}

Consent to Participate All authors are voluntarily participating for the submission of this research work.

Consent for Publication All authors expressed their consent to publish this research study.

Availability of data and material Data and material sharing not applicable.

Conflicts of Interest/Competing Interest The authors declare that they have no known competing financial interests or personal relationships that could have appeared to influence the work reported in this paper.

\section{Funding Statement}


This work is funded by BRNS (39/14/27/2016-BRNS/39064), Government of India. The authors sincerely thank BRNS for the same.

\section{Compliance with ethical standards}

Not applicable

Human and Animals Rights Authors declare no research involving human participants and/or animals was conducted.

\section{References}

1. Agarawal G. P, Fiber-Optic Communication System. New York, Wiley, 1997.

2. Zhang, Y.; Xiao, S.; Yu, Y.; Chen, C.; Bi, M.; Liu, L.; Zhang, L.; Hu, W. Experimental study of wideband inband fullduplex communication based on optical self-interference cancellation, Express, 24,3013930148, (2016).

3. Loyez, M.; Hassan, E.M.; Lobry, M.; Liu, F.; Caucheteur, C.; Wattiez, R.; DeRosa, M.C.; Willmore, W.G.; Albert, J. Rapid Detection of Circulating Breast Cancer Cells Using a Multiresonant Optical Fibre Aptasensor with Plasmonic Amplification, ACS sensors, 5, 454-463, (2050).

4. Leal-Junior, A.G.; Theodosiou, A.; Marques, C.; Pontes, M.J.; Kalli, K.; Frizera, A. Compensation method for temperature cross-sensitivity in transverse force applications with FBG sensors in POFs, J. Light. Technol., 36, 3660-3665, (2018).

5. Dong, y.; Chen, L.; Bao, X. High-spatial-resolution time-domain simultaneous strain and temperature sensor using Brillouin scattering and birefringence in polarization-maintaining fiber, IEEE Photon. Tech. Lett., 22, 1364-1366, (2010).

6. Frazao, 0.; Marques, L. M.; Baptista, J. M. Fiber Bragg grating interrogation based on highbirefringence fiber loop mirror for strain-temperature discrimination, Microwave Opt. Technol. Lett., 48, 2326-2328, (2006).

7. Nguyen, L.V.; Hwang, D.; Moon, D. S.; Chung, Y.; Simultaneous measurement of temperature and strain using a Lyot fiber filter incorporated with a fiber Bragg grating in a linear configuration, Sci. Technol., 20, 034006-1-5, (2009).

8. Uchimura, R.; Wada, A.; Tekuramori, S.; Takeuchi, M.; Tukida, O.; tanaka, S.; Takahasi, N. Simultaneous measurement of temperature and static strain using FBG Fabry-Perot interferometer in polarization-maintaining fiber, SPIE., 9157, (2014).

9. Xiong, L.; Zhang, D.; Li, L.; Guo, Y; EFPI-FBG hybrid sensor for simultaneous measurement of high temperature and strain, Opt. Lett., 12, 120605-1-5, (2014).

10. Liu, Q.; Ran, Z. L.; Rao, Y.J.; Shu-cheng, L.; Hui-Qin, Y.; Ya, H. Highly Integrated FP/FBG Sensor for Simultaneous Measurement of High Temperature and Strain, IEEE Photon. Tech. Lett., 26, 1715-1717, (2014). 
11. Ascorbe, J.; Coelho, L.; Santos, J. L.; Frazao, O.; Corres, J. M. Temperature Compensated Strain Sensor Based on Long-Period Gratings and Microspheres, IEEE Photon. Tech. Lett., 30, 67-70, (2018).

12. Esposito, F.; Srivastava, A; Campopiano, S.; ladicicco, A. Multi-parameter sensor based on single Long Period Grating in Panda fiber for the simultaneous measurement of SRI, temperature and strain, Opt Laser Technol., 113, 198-203, (2019).

13. Su, D.; Qiao, X.; Chen, F.; Bao, W. Compact Dual Fiber Bragg Gratings for Simultaneous Strain and High-temperature Measurement, IEEE Sens. J., 19,5660-5664, (2019).

14. Wu, Q.; Hatta, A.M.; Semenova, Y.; Farrell, G. Use of a single-multiple-single-mode fiber filter for interrogating fiber Bragg grating strain sensors with dynamic temperature compensation, Opt., 48, 5451-5458, (2009).

15. Kang, Z.; We, X.; Li, C.; Sun. J.; Wang, J.; Jian, S. Up-taper-based Mach-Zehnder interferometer for temperature and strain simultaneous measurement, Opt., 53, 2691-2695, (2014).

16. S.; Vasu, K.S.; Asokan, S.; Sood, A.K. Enhanced strain and temperature sensing by reduced graphene oxide coated etched fiber Bragg gratings, Opt. Lett., 41, 2604-2607, (2016).

17. Dey, K.; Roy, S.; P, Kishore.; M, Sai, Shankar.; B, Ramesh.; R, Ranjan.; Analysis and performance of edge filtering interrogation scheme for FBG sensor using SMS fiber and OTDR, Results in Optics, Volume 2, 100039, (2021).

18. Dey, K.; V, D, R, Pavan.; Roy, S.; M, Sai, Shankar.; B, Ramesh.; "Interrogation of SMS for measuring of temperature and strain using half-etched FBG with enhanced sensitivity," SPIE11355, MicroStructured and Specialty Optical Fibres VI, 113550Z, (2050).

19. Wu, Q.; Yuliya, S.; Hatta, A, M.; Wang, P.; Farrell, G.; "Single-mode-multimode-single-mode fiber structures for simultaneous measurement of strain and temperature", Microwave Opt. Technol. Lett., 53, 2181-2185, (2011).

20. Jin, W.; Michie, W.C.; Thursby, G.; Konstantaki, M.; Culshaw, B. Simultaneous measurement of strain and temperature: error analysis, Eng., 36, 598-609, (1997).

21. B, N, Shivananju.; M, Renilkumar.; G, R, Prashanth.; S, Asokan.; and M, M, Varma.; "Detection Limit of Etched Fiber Bragg Grating Sensors," Lightwave Technol. 31, 2441-2447 (2013).

\section{Table 1}


Table 1. Comparing the different sensing parameters with other reported methods

\begin{tabular}{|c|c|c|c|c|c|}
\hline $\begin{array}{c}\text { Temp. } \\
\text { Sensitivity } \\
\left(\mathrm{pm} /{ }^{\circ} \mathrm{C}\right)\end{array}$ & $\begin{array}{c}\text { Temp. } \\
\text { Range }\left({ }^{\circ} \mathrm{C}\right)\end{array}$ & $\begin{array}{c}\text { Strain } \\
\text { Sensitivity } \\
(\mathrm{pm} / \mu \varepsilon)\end{array}$ & $\begin{array}{c}\text { Strange } \\
\text { Range } \\
(\mu \varepsilon)\end{array}$ & Resolution & Ref. \\
\hline 10.39 & $0-80$ & 0.89 & $0-2000$ & $\pm 12 \mu \varepsilon, \pm 0.4^{\circ} \mathrm{C}$ & [4] \\
\hline 11.00 & $40-90$ & 1.22 & $0-1500$ & $\pm 6 \mu \varepsilon, \pm 0.5^{\circ} \mathrm{C}$ & {$[5]$} \\
\hline 8.40 & $(-) 70-20$ & 1.40 & $0-500$ & - & [7] \\
\hline 11.70 & $40-300$ & 1.20 & $0-500$ & - & [9] \\
\hline 47.00 & $20-60$ & 2.20 & $0-900$ & - & [10] \\
\hline 12.60 & $22-90$ & 1.14 & $0-800$ & $\pm 9.21 \mu \varepsilon, \pm 0.26^{\circ} \mathrm{C}$ & [11] \\
\hline 13.00 & $100-800$ & 3.25 & $0-1000$ & - & [12] \\
\hline 33.00 & $0-50$ & 0.74 & $0-708$ & $\pm 10.07 \mu \varepsilon, \pm 0.31{ }^{\circ} \mathrm{C}$ & [14] \\
\hline 9.70 & $10-60$ & - & $0-1667$ & $\pm 3.40 \mu \varepsilon, \pm 1{ }^{\circ} \mathrm{C}$ & [15] \\
\hline 20.00 & $(-) 100-300$ & 2.85 & $0-1000$ & - & [16] \\
\hline 20.30 & $25-225$ & 2.89 & $50-2050$ & $\pm 10 \mu \varepsilon, \pm 0.5^{\circ} \mathrm{C}$ & This Work \\
\hline
\end{tabular}

Figures

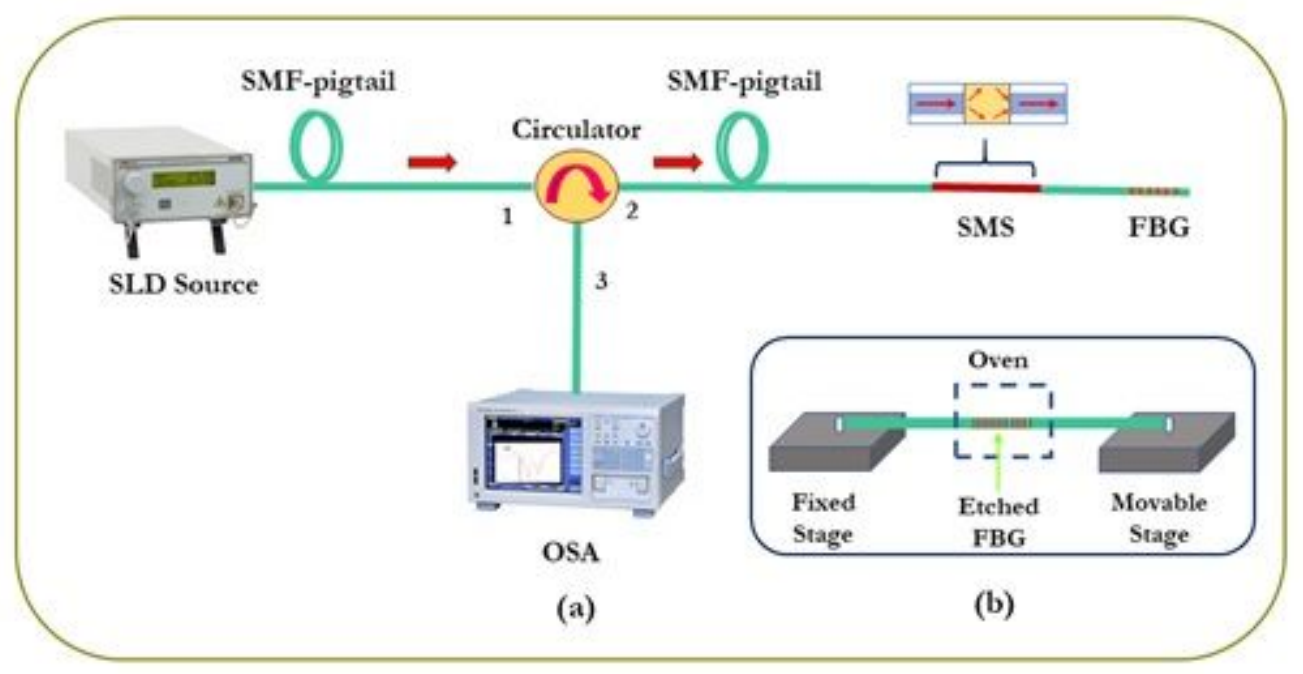


Figure 1

(a) Experimental setup for simultaneous measurement of strain and temperature and (b) Detailed schematic for strain and temperature characterization.
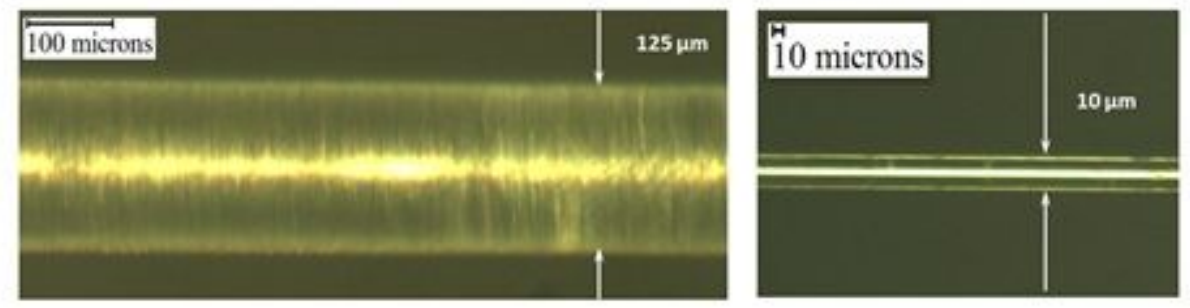

\section{Figure 2}

Microscopic image of (a) unetched FBG $(125 \mu \mathrm{m})$ and $(\mathrm{b})$ etched FBG $(10 \mu \mathrm{m})$.
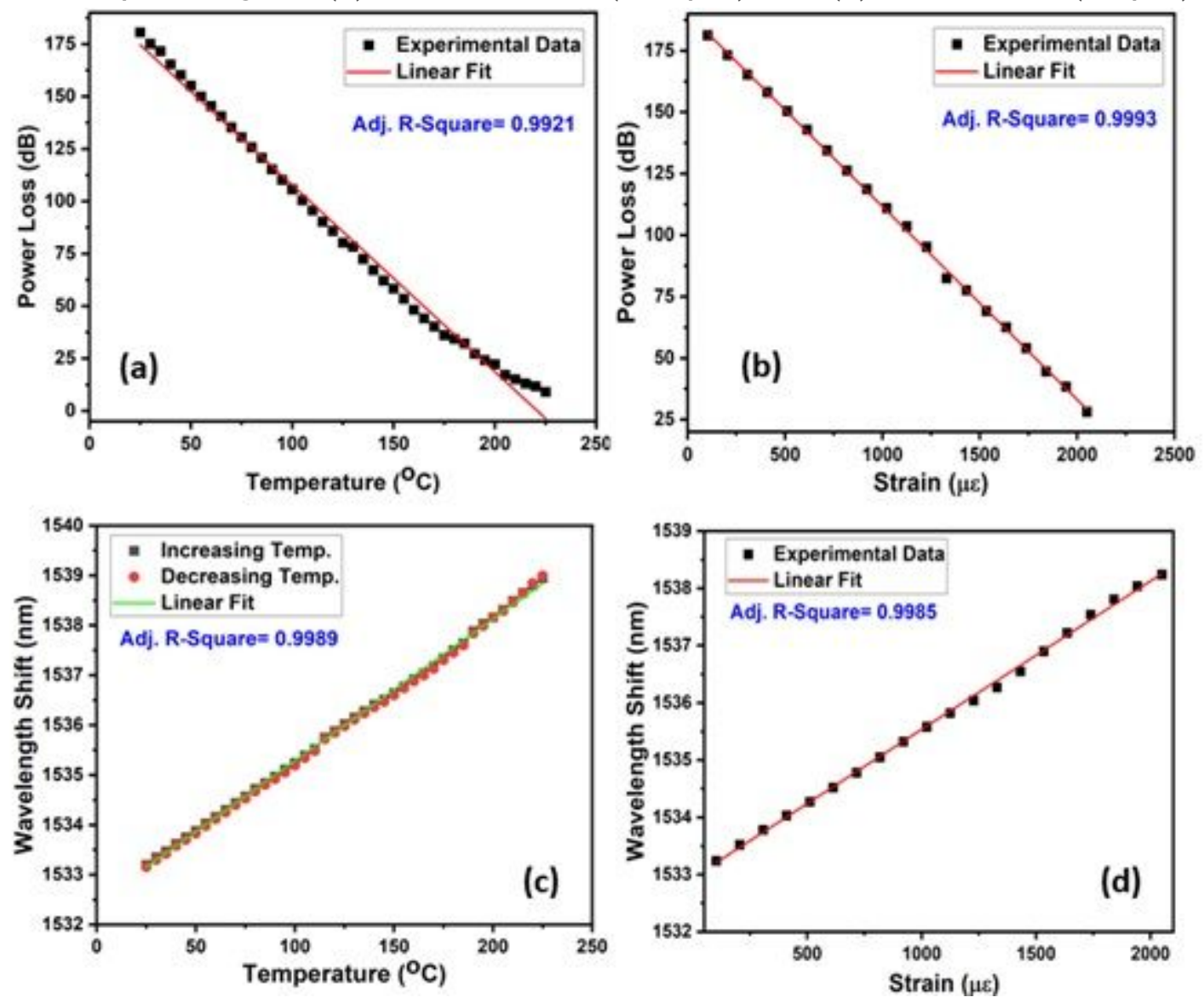

Figure 3

(a), (b) Change in the Optical power and (c), (d) Wavelength shift vs. variation in temperature and strain applied on FBG. 

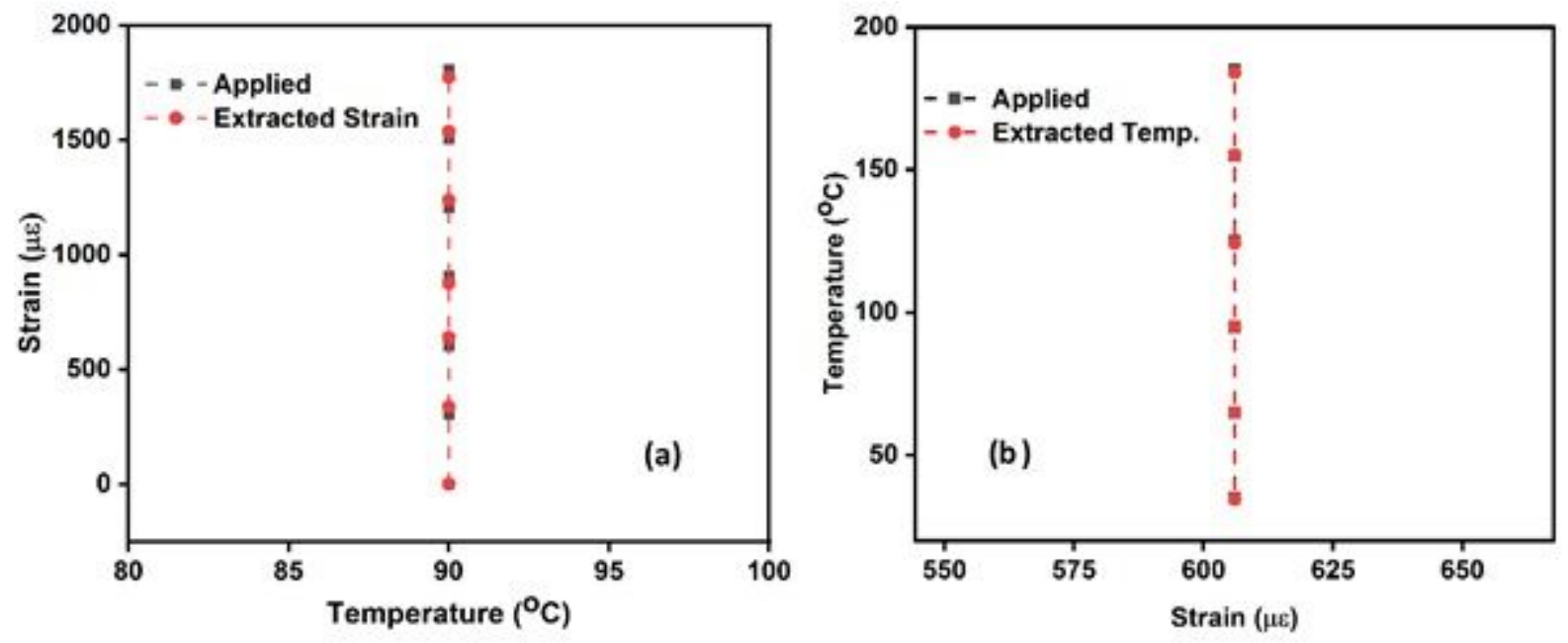

Figure 4

Sensor output for (a) applied strain at $90{ }^{\circ} \mathrm{C}$ and (b) the applied temperature at $606 \mu \rrbracket$.

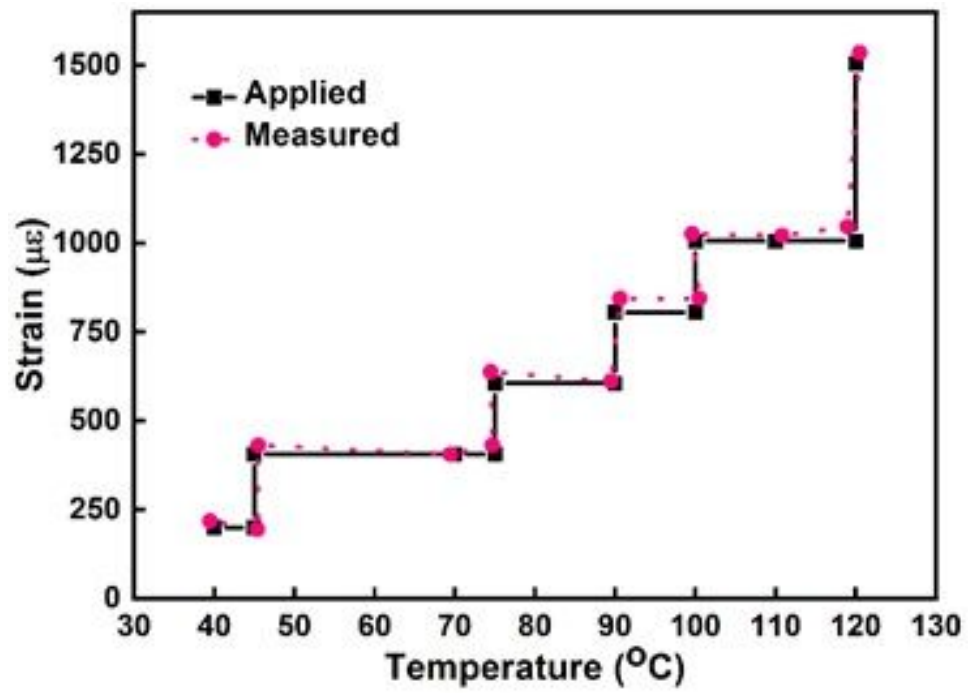

Figure 5

Experimental demonstration for calculating the temperature and strain simultaneously using the proposed sensor head. 